\section{MS7-P5 Application structures of nucleotide-protein complexes to study RNA recognition by bacterial and archaeal Lsm proteins}

Alexey D. Nikulin ${ }^{1}$, Natalia V. Lekontseva ${ }^{1}$, Victoria N. Murina ${ }^{1}$, Ekaterina Y. Nikonova ${ }^{1}$, Alice O. Mikhaylina ${ }^{1}$, Svetlana V. Tishchenko $^{1}$

1. Institute of Protein Research, Russia Academy of Sciences

email: nikulin@vega.protres.ru

Sm-like proteins (Lsm) exist in all three domains of life and are defined by the so-called Sm fold, which is comprised of an $\mathrm{N}$-terminal $\alpha$-helix and five anti-parallel $\beta$-strands. Bacterial Lsm proteins $\mathrm{Hfq}$ is a post-translational regulator of gene expression that binds small non-coding RNAs (sRNA) and promotes their interaction with mRNAs. Eukaryotic Lsm proteins act as chaperone for mRNAs and non-coding RNAs through various steps in metabolism. Function of the archaeal Lsm proteins have studied poor but it is known they bound archaeal sRNA.

Structures of Lsm proteins in complexes with short oligo-RNAs promote understanding involvement of the proteins in the RNA metabolism greatly. Recently we have used complexes of proteins with single ribonucleotides to determine the RNA-binding sites on the protein surface [1]. The complexes can be obtained before crystallization or by soaking of the protein crystals in the ribonucleotides solution. Typically, preparation of nucleotide-protein crystals is easier that the crystallization of large RNA-protein complexes. Using this technique we have identified three different RNA-binding sites on the Hfq surface, one of them have been located for the first time. Now we are using this technique to study archaeal Lsm proteins from Methanococcus jannaschii and Sulfolobus solfataricus and our last results will be demonstrated. This method can be used to study any RNA-binding protein interacting with single-stranded RNA.

This work was supported by the Russian Scientific Foundation (grant \# 14-14-00496) and by the Russian Foundation for Basic Research (grant \#13-04-00783).

[1] V. Murina, N. Lekontseva, A. Nikulin, Hfq binds ribonucleotides in three different RNA-binding sites, Acta Crystallogr, D69 (2013) 1504-1513.

Keywords: Lsm, Hfq, archaea, RNA-protein interaction, single-stranded RNA, ribonucleotide-protein complexes

\section{MS7-P6 Structural biology of 5hmC-specific endonuclease PvuRts1I}

Asgar Abbas Kazrani ${ }^{1}$, Monika Kowalska ${ }^{1}$, Honorata Czapinska ${ }^{1}$, Matthias Bochtler ${ }^{1,2}$

1. International Institute of Molecular and Cell Biology, Trojdena 4, 02109 Warsaw, Poland

2. Institute of Biochemistry and Biophysics, Polish Academy of Sciences, Pawinskiego 5a, 02106 Warsaw, Poland

email: abbas@iimcb.gov.pl

PvuRts1I is a prototype for a larger family of restriction endonucleases that cleave DNA containing 5-hydroxymethylcytosine $\quad(5 \mathrm{hmC})$ or 5-glucosylhydroxymethylcytosine $(5 \mathrm{ghmC})$, but not 5-methylcytosine $(5 \mathrm{mC})$ or cytosine. Here, we report a crystal structure of the enzyme at $2.35 \AA$ resolution. Although the protein has been crystallized in the absence of DNA, the structure is very informative. It shows that PvuRts1I consists of an N-terminal, atypical PD-(D/E)XK catalytic domain and a C-terminal SRA domain that might accommodate a flipped $5 \mathrm{hmC}$ or $5 \mathrm{ghmC}$ base. Changes to predicted catalytic residues of the PD-(D/E)XK domain or to the putative pocket for a flipped base abolish catalytic activity. Surprisingly, fluorescence changes indicative of base flipping are not observed when PvuRts1I is added to DNA substrates containing pyrrolocytosine in place of $5 \mathrm{hmC}(5 \mathrm{ghmC})$. Despite this caveat, the structure suggests a model for PvuRts1I activity and presents opportunities for protein engineering to alter the enzyme properties for biotechnological applications.

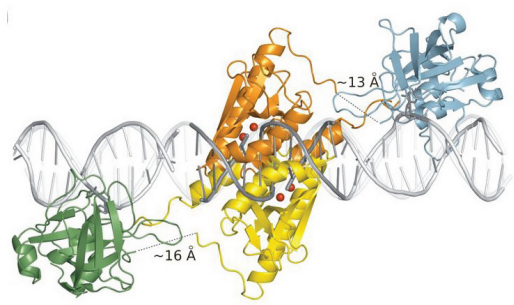

Figure 1. Model for PvuRts1I dimer binding to $5 \mathrm{hmC}$ containing DNA

Keywords: PvuRts1I, endonuclease, SRA, PD-(D/E)XK, 5-hydroxymethylcytosine 